\title{
Collisionality dependent transport in TCV SOL plasmas
}

\author{
O E Garcia ${ }^{1}$, R A Pitts ${ }^{2}$, J Horacek ${ }^{3}$, J Madsen ${ }^{1}$, V Naulin ${ }^{1}$, A H Nielsen ${ }^{1}$ \\ and J Juul Rasmussen ${ }^{1}$ \\ ${ }^{1}$ Association EURATOM-Risø National Laboratory, Technical University of Denmark, \\ OPL-128, PO Box 49, DK-4000 Roskilde, Denmark \\ ${ }^{2}$ Ecole Polytechnique Fédérale de Lausanne (EPFL), Centre de Recherches en Physique des \\ Plasmas, Association EURATOM-Confédération Suisse, CH-1015 Lausanne, Switzerland \\ ${ }^{3}$ Association EURATOM-Institute of Plasma Physics, Prague, Czech Republic \\ E-mail: odd.erik.garciaerisoe.dk
}

\begin{abstract}
Results are presented from probe measurements at the outboard midplane scrapeoff layer region of TCV current scan experiments. It is shown that with decreasing plasma current, or increasing magnetic connection length, the radial particle density profile becomes broader and the fluctuation levels and turbulence driven radial transport increases. In the far scrape-off layer the fluctuations exhibit a high degree of statistical similarity. Together with previous TCV density scan experiments, this strongly indicates that plasma fluctuations and radial transport increases with plasma collisionality. Such a collisionality dependence is consistent with a recent theory for radial blob motion, which suggests that filamentary structures become electrically disconnected from the target sheaths at large collisionality and thus experience less sheath dissipation. This increases the radial convective transport and is likely linked to the discharge density limit.
\end{abstract}




\section{Introduction}

The inherently fluctuating nature of the scrape-off layer (SOL) in magnetically confined plasmas has been known ever since the first electric probe measurements and has been found to prevail in virtually all magnetic geometries and confinement states [1-5]. Recent progress in experimental measurements and theoretical modelling indicate that the turbulence driven transport is largely mediated by filamentary structures which are elongated along the field lines and appear as blobs of excess particles and heat when viewed in the plane perpendicular to the field lines [6-10].

Probe measurements from a number of tokamak experiments have demonstrated that as the line-averaged plasma density $\bar{n}_{\mathrm{e}}$ increases, the radial particle density profile in the SOL becomes broader and plasma-wall interactions increase [11-15]. The particle density profile exhibit a two-layer structure. Close to the separatrix in the so-called near SOL it has a steep exponential decay and moderate fluctuation levels. Beyond this region, in the so-called far SOL, the profile has a much more gradual exponential decay and a fluctuation level of order unity. As the discharge density limit is approached, the break point moves radially inwards such that at the highest attainable plasma density, the far SOL profile extends all the way to the magnetic separatrix [11-15]. Recent measurements on TCV have demonstrated a high degree of statistical similarity in the region with broad plasma profiles. In particular, the radial variation of the relative fluctuation level, skewness and flatness moments of the plasma fluctuations and the temporal correlations are similar for all values of the line-averaged plasma density [16-20].

Associated with the broadening of the particle density profile are large fluctuation levels and turbulence driven radial transport. This is generally believed to be due to increased plasma collisionality $v_{\mathrm{e}}^{*}$, defined by $v_{\mathrm{e}}^{*}=L_{\|} / \lambda_{\mathrm{ei}}$, where $\lambda_{\mathrm{ei}}$ is the mean free path for electronion collisions. The plasma collisionality increases linearly with the plasma density and the magnetic connection length $L_{\|}$, which in the experiments are readily controlled through the line-averaged plasma density and the plasma current $I_{\mathrm{p}}$. Such a collisionality dependence of radial convective transport is likely linked to the empirical discharge limit, indicating that there is a maximum line averaged particle density and a minimum plasma current for which the frequency of disruptive terminations increases rapidly [21-25].

In this paper, an analysis of probe measurements at the outboard midplane region from a plasma current scan experiment on TCV is presented. These measurements demonstrate a broadening of the radial particle density profile and increased fluctuations and turbulence driven transport as the plasma current decreases. Similar to the density scan experiments, the fluctuations are found to exhibit a high degree of statistical similarity in the far SOL region. The experimental findings support the conjecture that for large plasma collisionality, filamentary plasma structures become electrically disconnected from the sheaths at the divertor targets. This results in a substantial enhancement of the radial convective transport. Interchange motions of plasma filaments thus appear as the salient mechanism underlying main chamber recycling, and the collisionality dependence is qualitatively consistent with the plasma density and current scaling of the discharge disruption limit. 
In the following section the physical mechanism for radial blob motion and the effect of sheath dissipation is described. In section 3 the experimental measurements are presented, and finally a discussion of the results and the conclusions drawn from them are given in section 4 .

\section{Radial interchange motion of plasma filaments}

Interchange motions driven by the non-uniform magnetic field has long been considered as a candidate mechanism to explain the large fluctuation level and turbulence driven transport in the SOL. Recent analytical and numerical work have demonstrated that an isolated blob-like structure can propagate a radial distance many times its initial size at large velocity [6-10]. The collective plasma motions are governed by the vorticity equation, which to lowest order can be written as

$$
\rho_{\mathrm{m}}\left(\frac{\partial}{\partial t}+\mathbf{V}_{E} \cdot \nabla\right) \frac{\nabla_{\perp}^{2} \phi}{B}+\frac{1}{B} \widehat{\mathbf{b}} \times(\boldsymbol{\kappa}+\nabla \ln B) \cdot \nabla P=\nabla \cdot \mathbf{J}_{\|},
$$

where $\rho_{\mathrm{m}}$ is the plasma mass density, $P$ the plasma pressure, $\boldsymbol{\kappa}=(\widehat{\mathbf{b}} \cdot \nabla) \widehat{\mathbf{b}}$ the magnetic field curvature vector and $\widehat{\mathbf{b}}$ the unit vector along the magnetic field. The first term on the left hand side of this equation describes plasma inertia through polarization currents. This predominantly involves the rate of change of the curl of the electrical drift $\mathbf{V}_{E}=\widehat{\mathbf{b}} \times \nabla \phi / B$, where $\phi$ is the electrostatic potential. The second term on the left hand side describes electric charge polarization due to guiding center drifts caused by the non-uniform magnetic field, while the right hand side describes the effect of parallel electric currents, $\mathbf{J}_{\|}$.

In the absence of parallel currents, the left hand side of equation (1) predicts an inertial velocity scaling for isolated blob-like structures given by

$$
\frac{C_{\mathrm{b}}}{C_{\mathrm{s}}} \sim\left(\frac{2 \ell}{R} \frac{\triangle P}{\Pi}\right)^{1 / 2}
$$

where $C_{\mathrm{s}}=\left(\Pi / \rho_{\mathrm{m}}\right)^{1 / 2}$ is the acoustic speed based on a homogeneous background pressure $\Pi, \ell$ is the structure size in the plane perpendicular to the magnetic field, $\triangle P / \Pi$ is the relative amplitude of the blob structure, and $R$ is the magnetic field radius of curvature. Twodimensional numerical simulations of the dynamical evolution of a blob-like structure initially at rest in a homogeneous plasma have demonstrated a fast radial acceleration, formation of a steep front and a trailing wake, and radial motion over a distance many times the initial structure size $[9,10]$. An example of this is presented in figure 1, showing the temporal evolution of the radial center of mass velocity. The signal recorded by a probe located at the symmetry axis a distance of $5 \ell$ in front of the initial blob position, also shown in figure 1 , reveals the presence of a steep front and a trailing wake. This simulation was performed for the experimentally relevant parameters $\mathrm{Ra}=2 C_{\mathrm{S}}^{2} \ell^{3} / \mu \chi R=10^{6}$ and $\operatorname{Pr}=\mu / \chi=1$, where $\mu$ and $\chi$ are the kinematic viscosity and thermal diffusion, respectively. The variation of the maximum radial center of mass velocity with collisional dissipation for $\operatorname{Pr}=1$ is presented in figure 2. For Ra larger than $10^{4}$ the inertial velocity scaling given by equation (2) prevails, and collisional transport across field lines does not significantly alter the transient radial motion of blob structures. Further description of the model and simulations can be found in $[9,10]$. 
Parallel currents will generally reduce the rate of charge polarization by magnetic guiding center drifts. For the special case of a collisionless plasma, perfectly field-aligned filamentary structures will be effectively connected to sheaths where the field line intersect material surfaces. At these surfaces the well-known sheath boundary condition prevails,

$$
J_{\|}=-e n C_{\mathrm{s}}\left[1-\exp \left(-\frac{e \phi}{T_{\mathrm{e}}}\right)\right] \widehat{\mathbf{n}} \cdot \widehat{\mathbf{b}},
$$

where $\widehat{\mathbf{n}}$ is the surface unit normal vector. Linearizing the exponential function and averaging the vorticity equation along magnetic field lines, the contribution of parallel currents yields the conventional sheath dissipation term,

$$
\left\langle\nabla \cdot \mathbf{J}_{\|}\right\rangle=\frac{e n C_{\mathrm{s}}}{L_{\|}} \frac{e\langle\phi\rangle}{T_{\mathrm{e}}} .
$$

In the vorticity equation this sheath dissipation acts preferentially on large spatial scale lengths, and results in a strong reduction of the maximum velocity and radial transport of filamentary structures. This is clearly demonstrated in figure 2, which presents the scaling of the maximum radial center of mass velocity with respect to the non-dimensional sheath dissipation coefficient, $\Lambda=2 C_{\mathrm{s}} \ell^{2} / \gamma L_{\|} \rho_{\mathrm{s}}^{2}$. Here $\gamma=\left(2 C_{\mathrm{S}}^{2} / \ell R\right)^{1 / 2}$ is the ideal interchange rate. For typical TCV SOL plasma parameters and a blob size of $1 \mathrm{~cm}$ and relative amplitude of 0.5 , the dimensionless sheath dissipation parameter $\Lambda$ is of order unity. Under collisionless conditions, sheath dissipation is thus expected to severely slow down the radial motion of plasma filaments.

The presence of ballooning in the fluctuation level and radial turbulence driven transport has been demonstrated in several tokamak experiments [26-30] As a consequence, particles and heat enter the SOL predominantly on the outboard midplane region, and are subsequently transported along the field lines to the divertor targets and across field lines to the main chamber walls. This poloidally asymmetric transport is generally believed to be the cause of field-direction independent parallel flows in SOL plasmas [29-32]. Due to this ballooning, filamentary plasma structures have a strong modulation along the magnetic field lines with amplitudes peaked at the outboard midplane. Electric charge polarization, driven by the magnetic guiding center drifts, is therefore also localized on the low field side. Collisional friction impedes parallel particle transport and, due to ballooning, therefore also the electrical connection of filamentary structures with the electrostatic sheaths at the targets. The role of sheath dissipation is thus expected to diminish with increasing plasma collisionality. Although the effect of collisional friction is not explicitly taken into account in the present model, figure 2 clearly shows that sheath dissipation substantially limits the radial velocity of filamentary structures and hence the convective transport.

\section{TCV current scan experiments}

A list of the TCV pulse numbers for the current scan experiments presented here are given in table 4. This comprises five pulses with the plasma current ranging from $220 \mathrm{kA}$ to $380 \mathrm{kA}$ in steps of $40 \mathrm{kA}$. All measurements were performed at a line-averaged density of 
approximately $\bar{n}_{\mathrm{e}}=4.2 \times 10^{19} \mathrm{~m}^{-3}$ and an axial magnetic field of $1.43 \mathrm{~T}$. The plasma shape was similar for all currents, with a lower single null divertor geometry. The separatrixmain chamber wall distance $\lambda_{\mathrm{SOL}}$ at the outer midplane varied from approximately $19 \mathrm{~mm}$ at the lowest to $27 \mathrm{~mm}$ at the highest current. For the purpose of presenting the results, a normalized radial coordinate $\rho=\left(r-r_{\text {sep }}\right) / \lambda_{\text {SOL }}$ is defined, which takes the values 0 and 1 at the separatrix position $r_{\text {sep }}$ and the wall intersection point of flux surfaces mapped from the probe location to the outside midplane. The fast reciprocating probe with a 5-tip head enters the plasma poloidally approximately halfway between the plasma midplane and the X-point, and measures fluctuations in the ion saturation current and the floating potential at a sampling rate of $6 \mathrm{MHz}$. A combination of floating potential measurements from two pins separated poloidally by $1 \mathrm{~cm}$ yields an estimate of the poloidal electric field and thus the radial electric drift. Further information about the experimental setup and measurement techniques can be found in [16-20].

The average value of the down-stream and up-stream connection lengths and the plasma collisionality at the near SOL position $\rho=0.25$ are given in table 4 . In the estimate for the plasma collisionality, a particle density of $n=1.5 \times 10^{19} \mathrm{~m}^{-3}$ and electron temperature of $T_{\mathrm{e}}=15 \mathrm{eV}$ have been used, which are characteristic values at this position for all pulses in the current scan. The connection length and the plasma collisionality changes by more than a factor of two in these experiments. The radial profile of the time-averaged particle density presented in figure 3 shows that as the plasma current decreases, the profile becomes broader and there is a substantial increase of the particle density at the wall radius. The profile scale length in the far SOL changes from approximately $2 \mathrm{~cm}$ at the largest to $4 \mathrm{~cm}$ at the lowest plasma current. The relative fluctuation level, also presented in figure 3, is roughly the same for all plasma currents, increases in the near SOL, and is approximately constant in the far SOL with broad profiles. The plasma fluctuations are characterized by large-amplitude bursts as demonstrated by the positive values of the skewness and flatness moments presented in figure 4. These moments are defined such as to vanish for a normal distribution. The similar radial variation of these higher order moments across the current scan further demonstrate the universality of plasma fluctuations in the TCV SOL [16-20].

The radial profile of the time-averaged floating potential $\bar{V}_{\text {f }}$ presented in figure 5 shows a very dramatic change with $I_{\mathrm{p}}$. At high currents, the radial variation of the potential is qualitatively similar to that expected from the sheath boundary conditions described by equation (3). In a simple one-dimensional equilibrium with no electric currents, the potential will have the same radial variation as the electron temperature which is a monotonically decreasing function of the radial coordinate. However, at intermediate and small currents there is a local maximum of the floating potential in the SOL. The radial location of the peak value shifts radially outwards and the width becomes gradually broader with decreasing plasma current. The potential profile is clearly not determined by the sheath boundary conditions alone at small plasma currents. From figure 5 it is also seen that the standard deviation of the estimated radial electric drift decreases with increasing radial coordinate and plasma current.

The significant increase in the plasma and radial velocity fluctuation levels with decreasing plasma current also leads to larger turbulence driven radial particle fluxes, as 
shown in figure 6. The particle flux density decreases with radial coordinate for all but the highest plasma currents. The nearly flat flux profiles in the far SOL indicate that the radial turbulence driven particle flux dominate over parallel losses. This is likely to be the cause of the broadening of the particle density profile seen in figure 3. Within the standard transport paradigm, the turbulence driven particle flux is often parameterized in terms of an effective diffusion coefficient, defined by $D_{\text {eff }}=\lambda_{n} \bar{\Gamma} / \bar{n}$, where $\lambda_{n}$ is the scale length of the particle density profile. However, this effective diffusivity has been shown to be a strongly depend on radial coordinate and $\bar{n}_{\mathrm{e}}$ for density scan experiments. This is also the case for the current scan experiments considered here. A parameterization in terms of an effective radial convection velocity, defined by $V_{\text {eff }}=\bar{\Gamma} / \bar{n}$, yields more robust and interesting features. As seen from figure 6 , the effective convective velocity increases with radial coordinate for high plasma currents and is roughly constant as function of radius for the smallest currents. In the far SOL with broad particle density profiles the effective velocity is approximately $100 \mathrm{~m} \mathrm{~s}^{-1}$ for all currents.

Fluctuations in the particle density, the radial velocity and the turbulence driven radial particle flux in the far SOL of TCV exhibit universal properties across a broad parameter range. The rescaled probability distribution function (PDF) of the particle density at the wall radius, presented in figure 7 , collapse to a common shape for all values of $I_{\mathrm{p}}$. The rescaled distribution is positively skewed and flattened with an exponential tail, reflecting the abundance of large amplitude bursts in the time series. The latter is further demonstrated by the conditionally averaged particle density signal, also presented in figure 7. Large-amplitude events in the particle density signals have an amplitude of roughly four times the root mean square value above the mean and a duration of approximately $25 \mu \mathrm{s}$. The wave form is characterized by a steep front and a trailing wake, qualitatively similar to that presented in figure 1. The PDFs of the estimated radial velocity also display a universal shape at the wall radius. As seen in figure 8, the rescaled distributions fall on top of each other, and have slightly positive skewness and flatness moments. A conditional average of the estimated radial velocity, with an amplitude threshold on the particle density given by $n-\bar{n}>2.5 n_{\mathrm{rms}}$, reveals an outwards directed velocity associated with the large amplitude bursts. As seen from figure 8 , this velocity increases with decreasing plasma current.

\section{Discussion and conclusions}

The results from TCV current scan experiments presented here show a remarkable similarity to previous density scan experiments. This includes a broadening of the radial particle density profile and a similar radial variation of the relative fluctuation level, the skewness and the flatness moments in the far SOL region with broad profiles. At the wall radius both the rescaled PDFs and conditional averages of the particle density fluctuations collapse into a common curve for all plasma currents. These rescaled distributions and wave forms presented in figure 7 are in fact indistinguishable from the corresponding curves for the density scan experiments [20]. The fluctuations are dominated by large amplitude bursts, resulting in positively skewed and flattened distributions. There is an asymmetric wave form with a steep 
front and a trailing wake which is associated with outwards propagating blob-like structures. Interchange motions of plasma filaments thus appear as the origin of the anomalous cross-field transport in the SOL [16-20].

Probe measurements at the outboard midplane SOL region of TCV indicate that plasma fluctuations and radial transport increase with plasma collisionality. The collisionality $v_{\mathrm{e}}^{*}$ is proportional to the plasma density and the connection length, and hence inversely proportional to the plasma current. Recent theories for radial interchange motions of plasma filaments provide a plausible interpretation for the collisionality dependence or the radial convective transport. Due to strong ballooning, plasma filaments appearing in the SOL are modulated along magnetic field lines with a peak amplitude at the outboard midplane. Since parallel motions are impeded at large collisionality, the structures will be electrically disconnected from the target sheaths at large plasma densities and small plasma currents. The correspondingly reduced sheath dissipation implies faster radial propagation, which is driven by the magnetic guiding center drifts primarily at the outboard midplane. This is consistent with the substantial increase in the conditionally averaged radial velocity associated with large amplitude events in the particle density at the wall radius with decreasing plasma current, presented in figure 8. It should also be noticed that the standard deviation of the estimated radial velocity increases with decreasing plasma current throughout the SOL, and that the floating potential does not follow the radial temperature variation as expected from the sheath boundary conditions at small currents.

In summary, experimental measurements and theoretical analysis of plasma fluctuations and transport in SOL plasmas strongly indicate that interchange motions of blob-like structures is the cause of broad particle density profiles and large relative fluctuation levels. Electrical disconnection from the target sheaths at large collisionality leads to a substantial increase of the radial convective transport, which enhances plasma-wall interactions and is likely linked to the discharge density limit.

$\mathrm{O}$ E G and $\mathrm{J} M$ have been supported with financial subvention from the Danish Agency for Science, Technology and Innovation. J H was supported by the Grant Agency of the Academy of Sciences of the Czech Republic under Grant No. A100430502. 


\section{References}

[1] Surko C M and Slusher R E 1983 Science 221817

[2] Wootton A J, Carreras B A, Matsumoto H, McGuire K, Peebles W A, Ritz Ch P, Terry P W and Zweben S J 1990 Phys. Fluids B 22879

[3] Nedospasov A V 1992 J. Nuclear Mater. 196-198 90

[4] Endler M 1999 J. Nuclear Mater. 266-269 84

[5] Zweben S J, Boedo J A, Grulke O et al 2007 Plasma Phys. Control. Fusion 49 S1

[6] Krasheninnikov S I 2001 Phys. Lett. A 283368

[7] D’Ippolito D A, Myra J R and Krasheninnikov S I 2002 Phys. Plasmas 9222

[8] Bian N H, Benkadda S, Paulsen J-V and Garcia O E 2003 Phys. Plasmas 10671

[9] Garcia O E, Bian N H, Naulin V, Nielsen A H and Rasmussen J J 2005 Phys. Plasmas 12090701

[10] Garcia O E, Bian N H and Fundamenski W 2006 Phys. Plasmas 13082309

[11] LaBombard B, Boivin R L, Greenwald M, Hughes J, Lipschultz B, Mossessian D, Pitcher C S, Terry J L, Zweben S J and Alcator Group 2001 Phys. Plasmas 82107

[12] Lipschultz B, LaBombard B, Pitcher C S and Boivin R 2002 Plasma Phys. Control. Fusion 44733.

[13] Lipschultz B, Whyte D and LaBombard B 2005 Plasma Phys. Control. Fusion 471559.

[14] Whyte D G, Lipschultz B L, Stangeby P C, Boedo J, Rudakov D L, Watkins J G and West W P 2005 Plasma Phys. Control. Fusion 471579.

[15] LaBombard B, Hughes J W, Mossessain D et al 2005 Nucl. Fusion 451658

[16] Graves J P, Horacek J, Pitts R A and Hopcraft K I 2005 Plasma Phys. Control. Fusion 47 L1

[17] Horacek J, Pitts R A and Graves J P 2005 Czechoslovak Journal of Physics 55271

[18] Garcia O E, Horacek J, Pitts R A, Nielsen A H, Fundamenski W, Graves J P, Naulin V and Rasmussen J J 2006 Plasma Phys. Control. Fusion 48 L1

[19] Garcia O E, Horacek J, Pitts R A et al 2007 J. Nuclear Mater. 363-365 575

[20] Garcia O E, Horacek J, Pitts R A et al "Fluctuations and transport in the TCV scrape-off layer" to appear in Nucl. Fusion

[21] Greenwald M 2002 Plasma Phys. Control. Fusion 44 R27

[22] Xu X Q, Nevins W M, Rognlien T D et al 2003 Phys. Plasmas 101773

[23] Antar G Y, Krasheninnikov S I, Devynck P et al 2005 Phys. Plasmas 12082503

[24] D’Ippolito D A and Myra J R 2006 Phys. Plasmas 13062503

[25] Guzdar P N, Kleva R G, Kaw P K et al 2007 Phys. Plasmas 14020701

[26] Terry J L, Zweben S J, Hallatschek K et al 2003 Phys. Plasmas 101739

[27] Kirnev G S, Budaev V P, Grashin S A, Khimchenko L N and Srytchev D V 2005 Plasma Phys. Control. Fusion $\mathbf{4 5} 459$

[28] Gunn J, Boucher C, Dionne M et al 2007 J. Nuclear Mater. 363-365 484

[29] LaBombard B, Rice J E, Hubbard A E et al 2004 Nucl. Fusion 441047

[30] LaBombard B, Rice J E, Hubbard A E et al 2005 Phys. Plasmas 1205611

[31] Pitts R A, Horacek J, Fundamenski W et al 2007 J. Nuclear Mater. 363-365 505

[32] Erents S K, Pitts R A, Fundamenski W, Gunn J P and Matthews G F 2004 Plasma Phys. Control. Fusion 461757 


\begin{tabular}{ccccc} 
Pulse number & $I_{\mathrm{p}}[\mathrm{kA}]$ & $L_{\|}(\rho=0.25)[\mathrm{m}]$ & $v_{\mathrm{e}}^{*}(\rho=0.25)$ & Symbol \\
\hline 28218 & 220 & 28 & 160 & $\boldsymbol{\Delta}$ \\
28217 & 260 & 22 & 130 & $\bullet$ \\
28221 & 300 & 18 & 105 & $\bullet$ \\
28222 & 340 & 15 & 85 & $\bullet$ \\
28223 & 380 & 13 & 75 & $\boldsymbol{\nabla}$ \\
\hline
\end{tabular}

Table 1. A list of the TCV pulses considered in this paper, showing the pulse number, the plasma current, the averaged connection length, the plasma collisionality, and the plot symbol and color code used in the subsequent figures. 

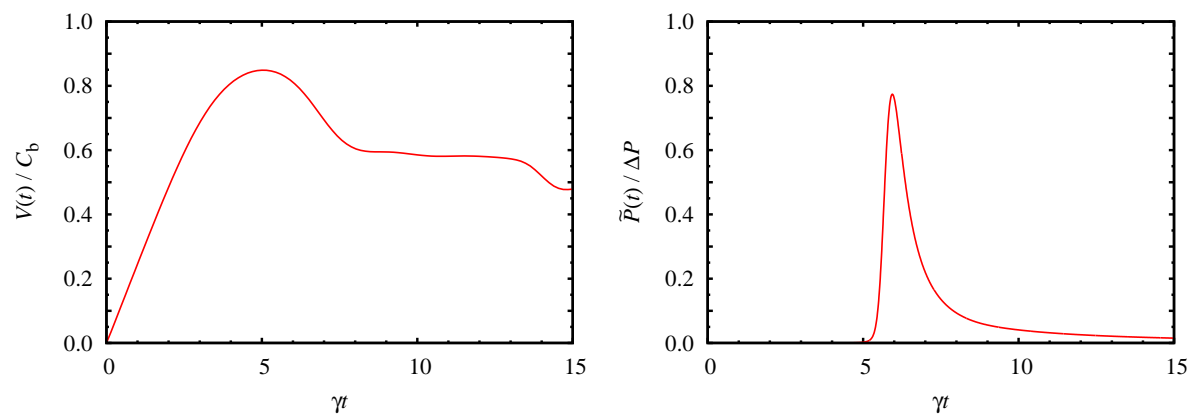

Figure 1. Temporal evolution of the maximum radial center of mass velocity for an isolated blob structure (left) and the pressure perturbation recorded by a probe located radially outwards by 5 times the initial structure size (right).
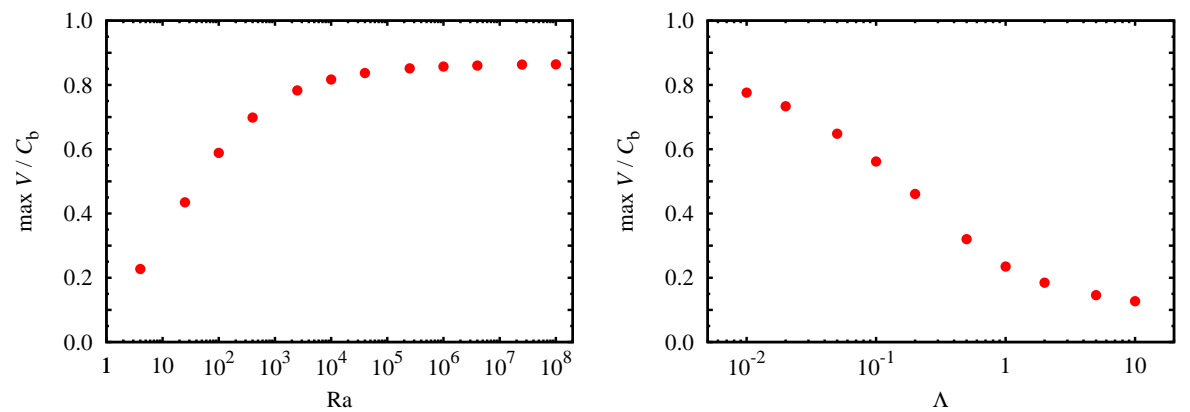

Figure 2. Scaling of the maximum radial center of mass velocity for an isolated blob structure as function of the effective bouyancy over collisional dissipation (left) and sheath dissipation (right).
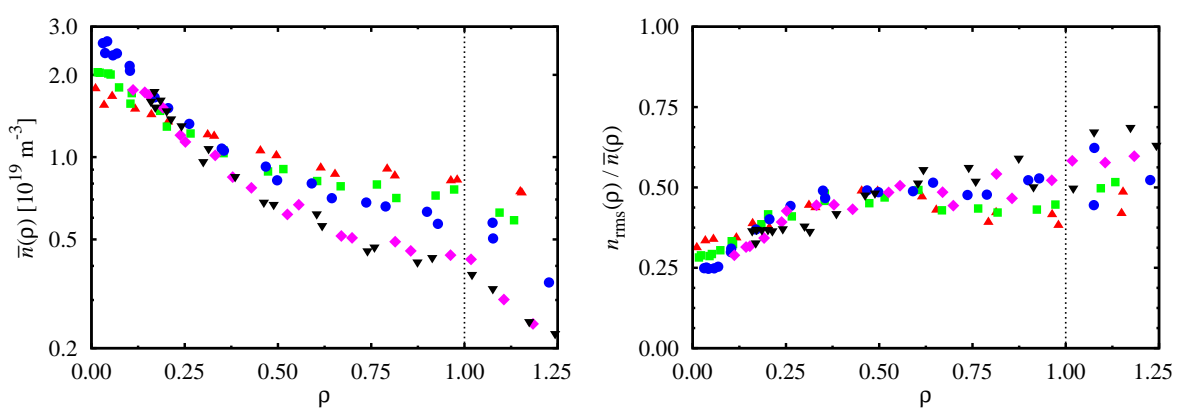

Figure 3. Radial profile of the average particle density (left) and the relative fluctuation level (right). 

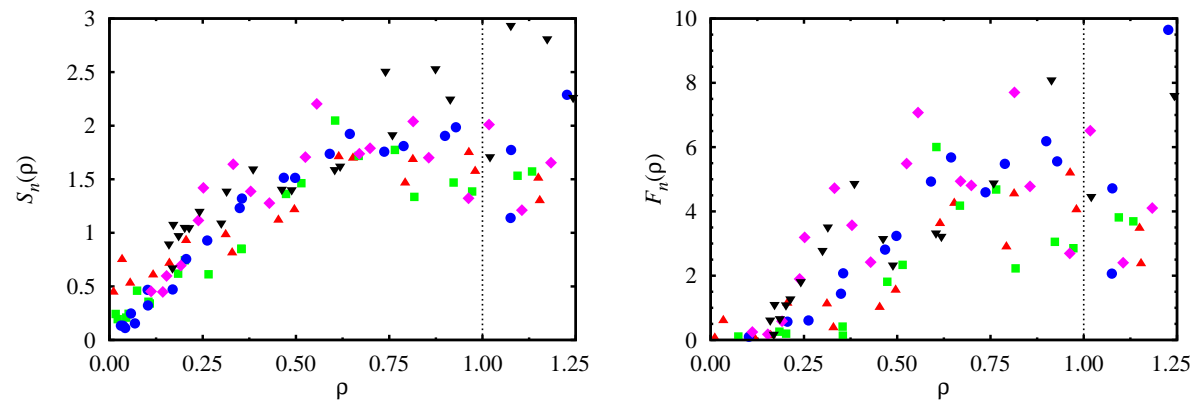

Figure 4. Radial profile of the skewness, $S_{n}$, (left) and the flatness, $F_{n}$, (right) moments of the particle density fluctuations.
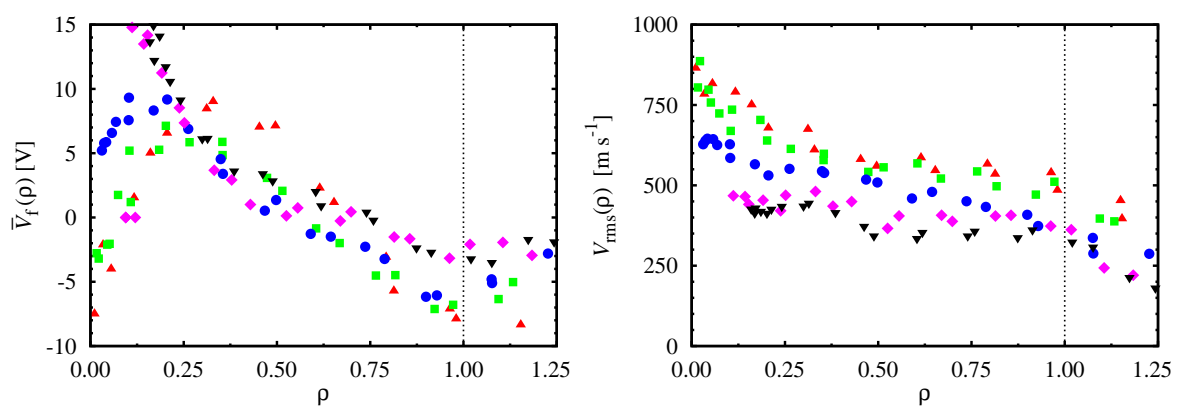

Figure 5. Radial profile of the average floating potential (left) and the relative fluctuation level of the estimated radial electric drift velocity (right).
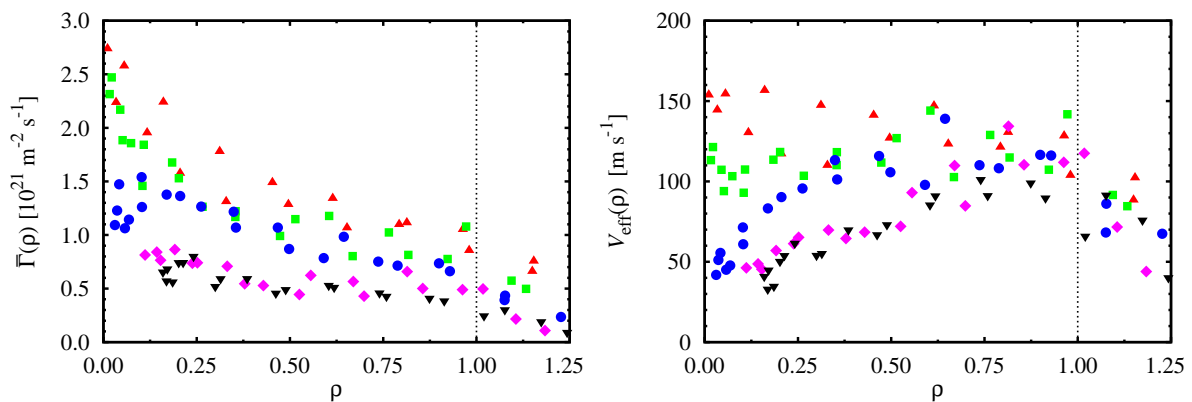

Figure 6. Radial profile of the estimated radial turbulence driven particle flux density (left) and the effective convection velocity (right). 

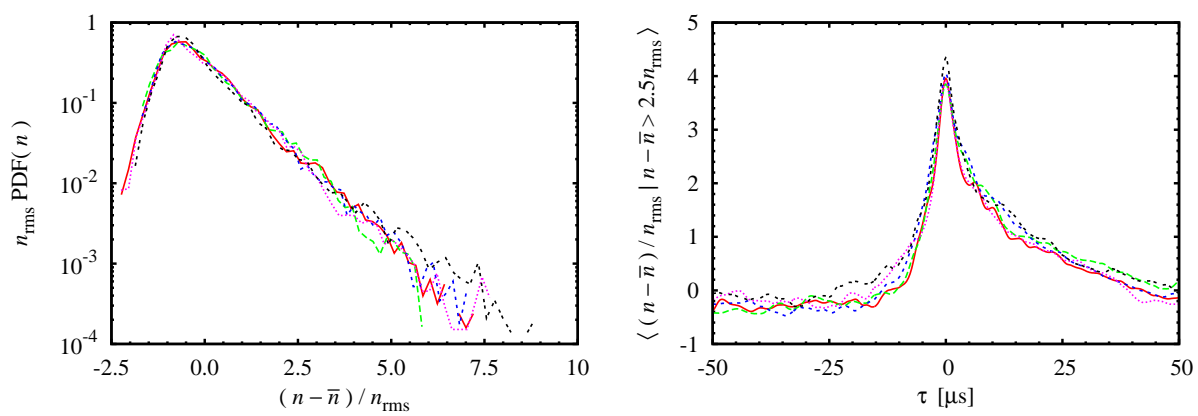

Figure 7. The PDF (left) and the conditionally averaged signal (right) for the particle density fluctuations at the wall radius.
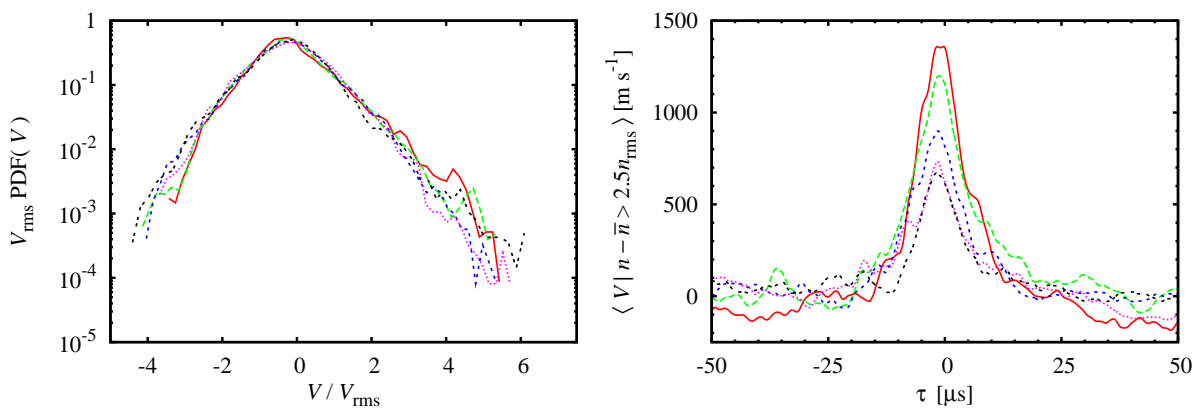

Figure 8. The PDF (left) and the cross-conditionally averaged signal (right) of the estimated radial electric drift velocity. 